\title{
Factors Affecting Survival in Patients with Colorectal Cancer in Shiraz, Iran
}

\author{
Mohammad Zare-Bandamiri ${ }^{1}$, Narges Khanjani ${ }^{2 *}$, Yunes Jahani ${ }^{3 \&}$, Mohammad \\ Mohammadianpanah ${ }^{4 \&}$
}

\begin{abstract}
Background: Colorectal cancer (CRC) is the third most common cancer in the world, and the fourth in Iran in both genders. The aim of this study was to find predictive factors for CRC survival. Materials and Methods: Medical records of 570 patients referred to the radiotherapy oncology department of Shiraz Namazi hospital from 2005 to 2010 were retrospectively analysed. Data were collected by reviewing medical records, and by telephone interviews with patients. Survival analysis was performed using the Cox's regression model with survival probability estimated with Kaplan-Meier curve. The log-rank test was used to compare survival between strata. Data was analyzed with Stata 12. Results: The five-year survival rate and the mean survival time after cancer diagnosis were $58.5 \%$ and $67 \pm 1.4$ months. On multivariate analysis, age of diagnosis, disease stage and primary tumor site, lymphovascular invasion and type of treatment (in colon cancer) were significant factors for survival. Conclusions: Age of diagnosis and type of treatment (adjuvant therapy in patients with colon cancer) were two modifiable factors related to survival of CRC patients. Therefore earlier diagnosis might help increase survival.
\end{abstract}

Keywords: Colorectal cancer - Iran - prognostic factors - survival

Asian Pac J Cancer Prev, 17 (1), 159-163

\section{Introduction}

Colorectal cancer (CRC) is the third most common type of cancer in the world and the fourth most common in Iran with an incidence rate of 5000 new cases every year, making up about $\% 8.12$ of all cases. In recent years, $\mathrm{CRC}$ has started to appear in younger populations with an incidence rate of two to six percent, and has resulted in the increased burden of this disease (Ferlay et al., 2010; Jemal et al., 2011; Torre et al., 2015).

Studies show that, although Iran is one of the countries with a low incidence rate of $\mathrm{CRC}$, but the number of new cases are increasing in this country (Health Deputy Minister of Health, 2007; Malekzadeh et al., 2009).

The survival rates associated with CRC vary across the world. The five-year survival rate for colon cancer is \% 44 globally. The five-year survival rates for colon cancer in developed, developing and Middle Eastern countries have been recorded as 46, 36, and 33 percent, respectively. But despite the increasing trend of CRC incidence, the mortality rates have declined and the fiveyear survival rates have increased. The growing survival rates in modern countries, and the variations in overall five-year survival throughout the world, suggest that determining predictive factors may improve the survival of CRC patients (Parkin et al., 2005; Aguero et al., 2012; Rezaianzadeh et al., 2015).

Previous studies mostly focused on demographic factors such as age and gender as well as tumor characteristics, including tumor size and stage. In the present study, we also examined lymphovascular invasion, proportion of involved lymph nodes as well as number of dissected lymph nodes during surgery and treatment methods. Moreover, this study is considered to be the most extensive study of colorectal cancer survival carried out in the south of Iran.

\section{Materials and Methods}

In this study, we reviewed demographic and clinical data of 570 colorectal cancer patients referred to the radiotherapy oncology department of Shiraz Namazi hospital from 2005 to 2010, Data were collected by reviewing medical records. We used death records and telephone interviews to find out the patients current situation or date of death. The age of diagnosis, gender of patients, number of dissected lymph nodes and positive lymph nodes (N-stage), tumor site, tumor stage, T-stage (based on the American Joint Committee on Cancer classification), tumor differentiation level, tumor size, 
type of treatment (adjuvant vs. neoadjuvant therapy), lymphovascular and perineural invasion were among the data collected in this study. Survival analysis was performed through Stata 12, using Cox's regression model. Survival probability was estimated by the KaplanMeier curves. The log-rank test was used to compare survival between strata of variables. In order to approve the proportional hazard rates a graph, with $-\mathrm{Ln}[-\mathrm{Ln}(\mathrm{St})$ on the $y$-axis and time on the $\mathrm{x}$-axis was used to test the proportional hazards regression model which is a key assumption of Cox proportional hazards models.

\section{Results}

Clinical and demographic characteristics of 570 patients with CRC are shown in table 1. More than half of the subjects were male (57.4\%). The mean age of diagnosis was $55.8 \pm 23.6$. Most patients were 50 to 70 years old $(45.5 \%)$, and the rectum was the primary cancer site in half

Table 1. Demographic Characteristics of the Colorectal Cancer Cases

\begin{tabular}{|c|c|}
\hline Variables & Mean \pm SD \\
\hline Age & $55.8 \pm 23.66$ \\
\hline Number of dissected lymph nodes & $8.81 \pm 6.42$ \\
\hline Number of positive lymph nodes & $5.61 \pm 2.23$ \\
\hline Tumor Size & $4.79 \pm 1.72$ \\
\hline Sex & Number (\%) \\
\hline Male & $327(57 \%)$ \\
\hline Female & $243(43 \%)$ \\
\hline Site of tumor & Number(percent) \\
\hline Rectum & $291(51 \%)$ \\
\hline Right and Transverse colon & $109(19 \%)$ \\
\hline Left colon & $53(9 \%)$ \\
\hline Sigmoid & $117(21 \%)$ \\
\hline \multicolumn{2}{|l|}{ T-stage } \\
\hline $\mathrm{T} 1$ & $7(1.2 \%)$ \\
\hline $\mathrm{T} 2$ & $111(19.2 \%)$ \\
\hline $\mathrm{T} 3$ & $421(73.1 \%)$ \\
\hline $\mathrm{T} 4$ & $31(5.5 \%)$ \\
\hline \multicolumn{2}{|l|}{$\mathrm{N}$-stage } \\
\hline No & $344(60 \%)$ \\
\hline N1 & $136(24 \%)$ \\
\hline $\mathrm{N} 2$ & $90(16 \%)$ \\
\hline \multicolumn{2}{|l|}{ Stage } \\
\hline I & $100(17.5 \%)$ \\
\hline II & $240(42.1 \%)$ \\
\hline III & $192(33.6 \%)$ \\
\hline IV & $38(6.8 \%)$ \\
\hline \multicolumn{2}{|l|}{ Grade } \\
\hline Well differentiated & $375(65.7 \%)$ \\
\hline Moderately differentiated & $159(27.8 \%)$ \\
\hline Poorly differentiated & $36(6.5 \%)$ \\
\hline \multicolumn{2}{|l|}{ Lymphovascular invasion } \\
\hline Yes & $212(37 \%)$ \\
\hline No & $358(63 \%)$ \\
\hline \multicolumn{2}{|l|}{ Perineural invasion } \\
\hline Yes & $161(28 \%)$ \\
\hline No & $409(72 \%)$ \\
\hline \multicolumn{2}{|l|}{ Treatment Method } \\
\hline Adjuvant therapy & $462(80.1 \%)$ \\
\hline Neoadjuvant therapy & $108(19.9 \%)$ \\
\hline
\end{tabular}

of these individuals. Approximately $80 \%$ of the patients underwent surgery before chemotherapy and radiotherapy. One third of the patients were registered living with stage 3 colorectal cancers $(33.6 \%)$ and the majority of patients were reported to have well differentiation cells $(65.7 \%)$.

The patients were followed until January 2015 and from 570 patients 232 had died $(40.7 \%)$. The mean survival time was $67 \pm 1.4$ months and the five-year survival rate was $56.9 \%$. In univariate analysis variables such as age of diagnosis, stage and primary tumor site, T-stage, N-stage, tumor differentiation level, proportion of involved lymph nodes, type of treatment, lymphovascular and perineural invasion were significant factors in survival. In the next step, variables with a P value less than 0.2 entered the multivariate regression model. Since tumor stage consists of two other variables including; $\mathrm{N}$-stage and T-stage, and given the collinearity between these two variables, thus, these two variables were excluded from multivariate Cox analysis. Age of diagnosis, tumor site, stage, lymphovascular invasion and type of treatment (in patient with colon cancer) were independent factors influencing survival of the CRC patients as shown in Table 2.

\section{Discussion}

Previous studies have revealed that, despite the lower incidence rate of CRC in comparison to other developed countries, there is an increasing trend of CRC incidence; as well, as YLL (years of life lost) in Iran (Safaee et al., 2012; Hoseini et al., 2014). This study investigated the factors affecting the survival of patients with CRC, and also investigated variables, that were not included in previous studies. The overall five-year survival rate in this study was $58.5 \%$. Previous colorectal cancer studies carried out in Iran reported that the five-year survival rates range from $31 \%$ to $68 \%$. However, Akhavan et al in Yazd, Iran (Akhavan et al., 2014), reported the survival rate of $83 \%$ for this cancer This result is probably due to exclusion of people with stage IV tumors from his study. O'Connell et al. (2004) in the US, reported the overall five-year survival rate of $65.2 \%$. The overall stage-specific 5-year survival

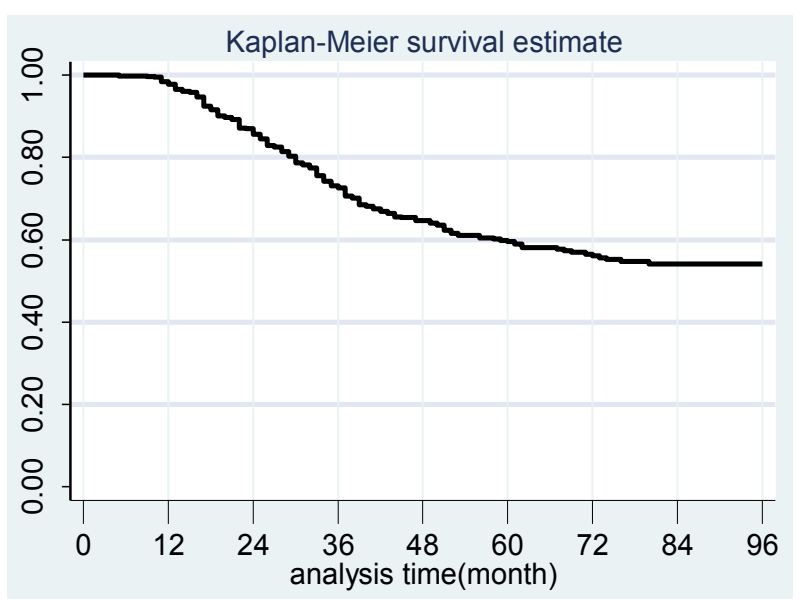

Figure 1. Survival Curve of Patients with Colorectal Cancer 
Factors Affecting Survival in Patients with Colorectal Cancer in Shiraz, Iran

Table 2. Univariate and multivariate analysis of prognostic factors in $\mathbf{5 7 0}$ patients with colorectal cancer

\begin{tabular}{|c|c|c|c|c|}
\hline \multirow[t]{2}{*}{ Variables } & \multicolumn{2}{|c|}{ Univariate } & \multicolumn{2}{|c|}{ Multivariate } \\
\hline & $\mathrm{HR}(95 \% \mathrm{CI})$ & P-Value & $\mathrm{HR}(95 \% \mathrm{CI})$ & P-Value \\
\hline \multicolumn{5}{|l|}{ Age } \\
\hline$<50$ year (reference) & 1 & 1 & & \\
\hline $50-70$ year & $2.28(0.93-1.74)$ & 0.118 & $1.15(0.84-1.58)$ & 0.36 \\
\hline$>70$ year & $2.11(1.49-3.01)$ & $<0.0001$ & $2.24(1.55-3.23)$ & $<0.0001$ \\
\hline \multicolumn{5}{|l|}{ Sex } \\
\hline Female (reference) & 1 & $-\cdots$ & & \\
\hline Male & $1.12(0.814-1.33)$ & 0.37 & ---- & \\
\hline \multicolumn{5}{|l|}{ Site of tumor } \\
\hline Rectum (reference) & 1 & 1 & & \\
\hline Right and transverse colon & $0.70(0.49-0.99)$ & 0.049 & $0.48(0.33-0.71)$ & $<0.0001$ \\
\hline Left colon & $0.44(0.25-0.77)$ & 0.004 & $0.40(0.23-0.71)$ & 0.002 \\
\hline Sigmoid & $0.71(0.51-1.00)$ & 0.056 & $0.62(0.44-0.98)$ & 0.009 \\
\hline \multicolumn{5}{|l|}{ Size of tumor } \\
\hline$\leq 5 \mathrm{~cm}$ (reference) & 1 & $-\ldots$ & & \\
\hline$>5 \mathrm{~cm}$ & $0.98(0.73-1.30)$ & 0.89 & $-\cdots$ & \\
\hline \multicolumn{5}{|l|}{ T-stage } \\
\hline $\mathrm{T} 1$ and T2 (reference) & 1 & $-\ldots$ & & \\
\hline $\mathrm{T} 3$ & $5.64(3.15-10.11)$ & $<0.0001$ & $-\ldots$ & \\
\hline $\mathrm{T} 4$ & $22.45(11.22-44.63)$ & $<0.0001$ & $-\ldots$ & \\
\hline \multicolumn{5}{|l|}{$\mathrm{N}$-stage } \\
\hline N0 (reference) & 1 & ---- & & \\
\hline N1 & $1.81(1.33-2.47)$ & $<0.0001$ & $-\ldots$ & \\
\hline N2 & $2.77(2.01-3.80)$ & $<0.0001$ & $-\cdots$ & \\
\hline \multicolumn{5}{|l|}{ Stage } \\
\hline I (reference) & 1 & 1 & & \\
\hline II & $3.62(1.98-6.61)$ & $<0.0001$ & $3.42(1.85-6.30)$ & $<0.0001$ \\
\hline III & $5.26(2.88-9.29)$ & $<0.0001$ & $3.54(1.81-6.91)$ & $<0.0001$ \\
\hline IV & $32.29(16.58-62.88)$ & $<0.0001$ & $35.63(12.26-73.54)$ & $<0.0001$ \\
\hline \multicolumn{5}{|l|}{ Grade } \\
\hline Well differentiated & 1 & 1 & & \\
\hline Moderately differentiated & $1.50(1.14-1.99)$ & 0.004 & $1.00(0.74-1.35)$ & 0.977 \\
\hline Poorly differentiated & $1.67(1.04-2.68)$ & 0.031 & $1.51(0.92-2.48)$ & 0.099 \\
\hline \multicolumn{5}{|l|}{ Dissected Lymph node } \\
\hline$>12$ (reference) & 1 & 1 & & \\
\hline$\leq 12$ & $1.73(0.97-3.10)$ & 0.062 & $1.10(0.58-2.02)$ & 0.761 \\
\hline \multicolumn{5}{|c|}{ Proportion of positive Lymph nodes } \\
\hline$\leq 0.16$ & 1 & 1 & & \\
\hline$>0.16$ & $2.09(1.61-2.71)$ & $<0.0001$ & $1.46(1.02-2.08)$ & 0.036 \\
\hline \multicolumn{5}{|l|}{ Lymphovascular invasion } \\
\hline No (reference) & 1 & 1 & & \\
\hline Yes & $2.63(2.03-3.41)$ & $<0.0001$ & $1.97(1.42-2.74)$ & $<0.0001$ \\
\hline \multicolumn{5}{|l|}{ Perineural invasion } \\
\hline No (reference) & 1 & 1 & & \\
\hline Yes & $1.79(1.38-2.33)$ & $<0.0001$ & $1.05(0.75-1.48)$ & 0.74 \\
\hline \multicolumn{5}{|l|}{ Treatment(colon) } \\
\hline Adjuvant therapy & 1 & 1 & & \\
\hline Neoadjuvant therapy & $2.02(1.25-3.29)$ & 0.004 & $2.76(1.57-4.81)$ & $<0.0001$ \\
\hline \multicolumn{5}{|l|}{ Treatment(rectum) } \\
\hline Adjuvant therapy & 1 & 1 & & \\
\hline Neoadjuvant therapy & $1.45(0.995-2.17)$ & 0.053 & $1.31(0.82-2.09)$ & 0.246 \\
\hline
\end{tabular}

rates in their study were $93.2 \%$ for stage I, and $8.1 \%$ for stage IV. In our study CRC was more common in men than in women which was compatible with the age-specific incidence rates reported by the Iranian Health Ministry in 2007(Health Deputy Minister of Health, 2007).

In this study, the most common tumor site was the rectum followed by the sigmoid. These findings were in line with some of the previous studies done in Iran and, the US and China (Bafandeh et al., 2008; Aguero et al., 2012; Yuan et al., 2013), However, Safaee in Iran (Azadeh et al., 2007; Safaee et al., 2012), reported that, in $62.9 \%$ of their patients, the most common site of CRC was the colon. Their different results can be due to a referral bias in our study; Our cases were from a radiotherapy center. Radiotherapy is an essential treatment for rectum cancer cases, but is recommended for only some colon cancer patients and according to their status.

This study, also showed that the survival rate associated with rectum cancer was lower than other sites. Yuan et al. (2013) in China, reported that the 3-year survival rate 
in rectum cancer patients was higher than colon cancer patients, while the 5-year survival rates among rectum cancer patients was lower than colon cancer patients (Yuan et al., 2013).

Few studies have ever studied tumor size in relation to survival rate in CRC (Moghimi-Dehkordi et al., 2008; Majek et al., 2012). In the present study, there were no significant relationship between tumor size and overall survival rates. However, the survival rates of patients with tumors bigger than $5 \mathrm{~cm}$ were a bit higher than patients with tumors smaller than $5 \mathrm{~cm}$. This could be due to the fact that, the tumors in the right and left colon (with average sizes of 5.7 and $4.7 \mathrm{~cm}$, respectively) have higher survival rates than rectum tumors (with average sizes of $4.5 \mathrm{~cm}$ ). Bafandeh et al in Tabriz, Iran showed that the average size of polyps in patients with colon polyps was bigger than patients with rectum polyps (Bafandeh et al., 2008).

By using univariate analysis, we found a significant relationship between T-stage and overall survival rates. Since the disease stage is highly influenced by T-stage (depth of bowel wall invasion), the variable, T-stage was not used in multivariate analysis. Moghimi-Dehkordi et al in Tehran, Iran, also suggested a significant relationship between T-stage and survival rates of CRC patients (Moghimi-Dehkordi et al., 2008). However, the collinearity between T-stage and $\mathrm{N}$-stage was not discussed in their study. It has been reported in the majority of studies that, tumor stage can independently affect the survival rate of CRC patients (Aguero et al., 2012; Majek et al., 2012).

In the present study, tumor grade (well, moderate or poorly differentiated) was a significant factor affecting the survival of CRC patients, while multivariate analysis showed no significant relationship between survival rates and tumor grade. This variable was significant in both univariate and multivariate analysis in Tehran (Moghimi-Dehkordi et al., 2008) too. In Yuan's study, after grade showed to be significant in univariate analysis, these variables were entered into multivariate analysis in two ways: First without entering tumor stage and then by entering the tumor stage. The result of this study in multivariate analysis showed that when tumor stage was not entered, grade was significant and when tumor stage was entered, grade was not significant (Yuan et al., 2013).

In the present study, the number of lymph nodes was significant in univariate analysis, but not in multivariate analysis. In this study lymph node stage was determined based on the American Joint Committee on Cancer classification, the 7 th edition, and showed that as the number of lymph nodes involved increased, the survival rate decreased. However in our study N-stage was not included in multivariate analysis due to its collinearity with disease stage.

Moreover, the ratios of positive lymph nodes to the total number of lymph nodes ( $\mathrm{pLNR}$ ) were calculated, and the mean of pLNR was 0.16 in our study. The survival rate was higher in patients with pLNR lower than 0.16. Ghahramani in Shiraz, Iran, found that the mean pLNR was 0.5 for rectal cancers and 0.37 for colon cancers respectively (Ghahramani et al., 2013). Mouge et al in UK, found that, the number of positive lymph nodes based on AJCC standards, was a significant factor in univariate analysis, but not in the multivariate analysis. Also in Mouge's study, the ratio of positive lymph nodes to the total dissected number of lymph nodes was a significant factor in both univariate and multivariate analysis. However, in their study this ratio was classified into four categories, less than $0.05,(0.05-0.19),(0.2-0.39)$ and (0.4-1) (Moug et al., 2011). In the present study, we classified this ratio into two categories, less than 0.16 and 0.16 or higher.

CRC treatment varies based on the tumor site. This study also looked at the relation between different treatments on survival among both rectum and colon cancer patients. Adjuvant therapy showed better survival rates in colon cancer patients, while the type of treatment was not significantly related to survival in rectum cancer patients. Moghimi-Dehkordi, showed that, survival rates were improved among patients who primarily underwent surgery. However, unlike findings in this study, surgery was not significant in multivariate analysis (MoghimiDehkordi et al., 2008). Akhoond et al. (2010) in Tehran, Iran, stated that, there were no significant relationships between primary treatment and CRC survival; however, primary treatment had a significant relationship with rectum cancer survival rate (Akhoond et al., 2010). Mehrabani et al. (2012) in Tehran, Iran, proposed that, treatment is an independent factor in survival and a combination of surgery, chemotherapy and radiotherapy, can produce remarkable results which increase the survival of patients in comparison to single therapy (Mehrabani et al., 2012). The findings of this study suggest that, surgery as the primary mode of treatment before chemotherapy and radiotherapy will lead to higher survival rates among CRC patients.

Findings of this study suggest that, factors such as; age of diagnosis, site, stage of tumor and lymphovascular invasion are most likely to have an effect on the survival rates of CRCs. Furthermore, adjuvant therapy in colon cancer patients increased the overall survival rate among these individuals and earlier diagnosis and appropriate treatment might help increase survival.

\section{References}

Aguero F, Murta-Nascimento C, Gallen M, et al (2012). Colorectal cancer survival: results from a hospital-based cancer registry. Rev Esp Enferm Dig, 104, 572-7.

Akhavan A, Binesh F, Soltani A (2014). Survival of rectal cancer in Yazd, Iran. Asian Pac J Cancer Prev, 15, 4857-60.

Akhoond MR, Kazemnejad A, Hajizadeh E, et al (2010). Comparison of influential factors affecting survival of patients with colon and rectum cancer using competing risks model. Koomesh, 12, 119-28.

Azadeh S, Moghimi-Dehkordi B, Fatem S, et al (2007). Colorectal cancer in Iran: an epidemiological study. Asian Pac J Cancer Prev, 9, 123-6.

Bafandeh Y, Khoshbaten M, Eftekhar Sadat AT, et al (2008). Clinical predictors of colorectal polyps and carcinoma in a low prevalence region: results of a colonoscopy based study. World J Gastroenterol, 14, 1534-8. 
Ferlay J, Shin H, Bray F, et al (2010). Estimates of worldwide burden of cancer in 2008:

GLOBOCAN (2008). Int J Cancer, 127, 2893-917.

Ghahramani L, Moaddabshoar L, Razzaghi S, et al (2013). Prognostic value of total lymph node identified and ratio of lymph nodes in resected colorectal cancer. Ann Colorectal Res, 1, 81-91.

Health Deputy Minister of Health NDMD, Cancer Center (2007) National report on cancer registration.

Hoseini S, Moaddabshoar L, Hemati S, et al (2014). An overview of clinical and pathological characteristics and survival rate of colorectal cancer in Iran. Ann Colorectal Res, 2, 17264.

Jemal A, Bray F, Center MM, et al (2011). Global cancer statistics. CA Cancer J Clin, 61, 69-90.

Majek O, Gondos A, Jansen L, et al (2012). Survival from colorectal cancer in Germany in the early $21^{\text {st }}$ century. British J Cancer, 106, 1875-80.

Malekzadeh R, Bishehsari F, Mahdavinia M, et al (2009). Epidemiology and molecular genetics of colorectal cancer in iran: a review. Arch Iran Med, 12, 161-9.

Mehrabani D, Almasi-Hashiani A, Moshfeghi K, et al (2012). Survival rate and its predictors in colorectal cancer patients in Southern Iran. Middle East J Sci Res, 12, 1072-77.

Moghimi-Dehkordi B, Safaee A, Zali MR (2008). Prognostic factors in 1,138 Iranian colorectal cancer patients. Int $J$ Colorectal Dis, 23, 683-8.

Moug SJ, McColl G, Lloyd SM, et al (2011). Comparison of positive lymph node ratio with an inflammation-based prognostic score in colorectal cancer. Br J Surg, 98, 282-6.

O'Connell JB, Maggard MA, Ko CY (2004). Colon cancer survival rates with the new american joint committee on cancer sixth edition staging. J National Cancer Ins, 96, 1420-5.

Parkin DM, Bray F, Ferlay J, et al (2005). Global cancer statistics, 2002. CA Cancer J Clin, 55, 74-108.

Rezaianzadeh A, Safarpour AR, Marzban M, et al (2015). A systematic review over the incidence of colorectal cancer in Iran. Ann Colorectal Res, 3, 25724.

Safaee A, Fatemi SR, Ashtari S, et al (2012). Four years incidence rate of colorectal cancer in Iran: a survey of national cancer registry data - implications for screening. Asian Pac J Cancer Prev, 13, 2695-8.

Torre LA, Bray F, Siegel RL, et al (2015). Global cancer statistics, 2012. CA Cancer J Clin, 65, 87-108.

Yuan Y, Li MD, Hu HG, et al (2013). Prognostic and survival analysis of 837 Chinese colorectal cancer patients. World $J$ Gastroenterol, 19, 2650-9. 